\title{
Bicalutamide increases phospho-Akt levels through Her2 in patients with prostate cancer
}

\author{
Claudio Festuccia ${ }^{1}$, Giovanni Luca Gravina ${ }^{2}$, Paola Muzi ${ }^{1}$, Roberto Pomante ${ }^{3}$, \\ Luca Ventura ${ }^{4}$, Robert $L$ Vessella ${ }^{5}$, Carlo Vicentini ${ }^{2}$ and Mauro Bologna ${ }^{1,6}$
}

\author{
Departments of ${ }^{1}$ Experimental Medicine, ${ }^{2}$ Surgery, University of L'Aquila, 67100 L'Aquila, Italy \\ ${ }^{3}$ Pathology Departments of G Mazzini Hospital, 68100 Teramo, Italy \\ ${ }^{4}$ San Salvatore Hospital, 67100 L'Aquila, Italy \\ ${ }^{5}$ Department of Urology, University of Washington, Seattle, Washington 98195, USA \\ ${ }^{6}$ Department of Basic and Applied Biology, University of L'Aquila, 67100 L'Aquila, Italy \\ (Correspondence should be addressed to C Festuccia, Dipartimento di Medicina Sperimentale, Cattedra di Patologia Generale, \\ Università dell'Aquila, Via Vetoio, Coppito 2, 67100 L'Aquila, Italy; Email: festucci@univaq.it) \\ $C$ Festuccia and $G \mathrm{~L}$ Gravina contributed equally to this work
}

\begin{abstract}
Bicalutamide monotherapy is emerging as an alternative in the treatment of locally advanced prostate cancer. However, a significant number of these patients will recur and be in need of second-line therapies. The knowledge of molecular arrangements after pharmacological therapy seems to be a new primary prerequisite to predict the efficacy or the failure of a secondary therapy. Based on these considerations, we have conducted this study in order to analyze the expressions of phosphatase and tensin homolog deleted on chromosome 10 (PTEN), Akt, epidermal growth factor receptor (EGFR), phospho-EGFR (p-EGFR), human EGFR2 (Her2), and phospho-Her2 (p-Her2) after bicalutamide treatment. For this purpose, we evaluated retrospectively 69 prostate cancer tissues derived from patients who received radical prostatectomy as the only treatment, and 81 from patients who received bicalutamide for 120 days before surgery. In addition, we analyzed at different time points the effects of castration performed on athymic mice bearing the LuCaP 35 xenograft line at different times. We observed that bicalutamide treatment increased significantly the levels of p-Akt, EGFR, and Her2 with a concomitant reduction in PTEN. This effect was time dependent and required of sufficient time to be evident as indicated by data obtained with the LuCaP 35 tumors. A logistic multiple regression analysis revealed that a switch of $p$-Akt control from a PTEN/EGFR- to Her2-after bicalutamide treatment was possible. Since Akt and Her2 can be associated with reduced drug sensitivity, our report suggests that the evaluation of molecular arrangements after bicalutamide treatment could be useful to identify subsets of patients who will be molecular permissive for new adjuvant anti-target therapies.
\end{abstract}

Endocrine-Related Cancer (2007) 14 601-611

\section{Introduction}

The benign prostatic epithelium as well as most prostatic adenocarcinomas (PCa) is dependent on androgens for the maintenance of proliferative potential as well as the inhibition of apoptosis. In fact, the dependence on androgens is the basis for androgen ablation therapy, the standard therapy for metastatic prostatic adenocarcinoma for the past 50 years. Unfortunately, the beneficial actions of existing endocrine measures are, in part, counteracted by the capacity of the tumor cells to eventually circumvent the use of steroid hormones, allowing them to continue to grow and progress despite such therapy. In fact, after a positive initial response, some tumors recur in an androgen-independent (AI) form. For these reasons, additional targets and therapies are needed in conjunction with androgen ablation. On the other hand, identification of new biomarkers is needed to identify high-risk patients as candidates for new (adjuvant) therapies and to find new surrogate endpoints to assess the efficacy of these treatments (Unni et al. 2004). 
Although the molecular mechanisms underlying the adaptative phenomenon after an androgen deprivation therapy have not been elucidated, accumulating evidence indicates that PI3K/Akt signaling pathway is involved (Ayala et al. 2004, Lee et al. 2004, Uzgare \& Isaacs 2004, Shukla et al. 2005, Chen et al. 2006). Activation of PI3K leads to increased Akt phosphorylation and activation. In turn, activated Akt inhibits prostate cancer cell suicide by blocking many of the key components of the suicide 'machinery'. Although other mechanisms, such as autocrine growth factor loops, may contribute to activation of PI3K/Akt in PCa cells, about $30 \%$ of prostate cancers exhibit phosphatase and tensin homolog deleted on chromosome 10 (PTEN), a potent tumor suppressor gene. Mutations and the loss of PTEN (Whang et al. 1998, Hermans et al. 2004, Bertram et al. 2006, Verhagen et al. 2006) leads to constitutive activation of the Akt pathway providing a mechanism whereby prostate tumor cells survive after the withdrawal of exogenous trophic factors or androgens. In addition, there are multiple lines of evidence indicating that the EGFR and HER2/neu are signaling molecules that control many aspects of tumor cell behavior-inducing changes in the survival rate. In addition, high levels of EGFR and HER2/neu can be demonstrated in PCa, especially after hormone therapy (Osman et al. 2001, Shi et al. 2001, Di Lorenzo et al. 2002, Gravina et al. 2004). The increased expression and activation of these receptors start a signaling cascade resulting in tumor proliferation and cell adhesion and invasion (Craft et al. 1999, Gross et al. 2004, Festuccia et al. 2005a).

Little attention has been paid to the impact of 'biological behavior' indicators on prostate cancer after bicalutamide treatment. Presently, bicalutamide is administered exclusively on the basis of clinical information. However, knowledge of the molecular profile after treatment might be critical considering its large-scale use in clinical practice (Kroger et al. 2006). The aim of this work was to evaluate retrospectively if bicalutamide treatment can induce molecular arrangements in PCa tumors when administered in a relatively short-term therapy as can be the NHT. We included in our study only subjects who had an operable PCa with intermediate- to high-risk characteristics. Therefore, no recurrent $\mathrm{PCa}$ cases were included. The only recurrent tumor was present in the $\mathrm{LuCaP} 35$ xenograft model that was studied after short term ( $0-7$ days) of castration and in the recurrent phase.

This is the first report in which it is demonstrated that bicalutamide administered in a neoadjuvant hormone therapy (NHT) regimen, and thus as a relatively short time of treatment, results in incremental Akt phosphorylation and activity. This could be determined by a switch of its control from a predominant Akt activation by PTEN loss/reduction and EGFR activation to a strong Her2mediated Akt activation. We demonstrate that this adaptative phenomenon requires a sufficient time to fully develop. From the LuCaP 35 studies which involved 7 days of monitoring this signaling pathway postcastration, Her2 and PTEN were altered almost immediately, whereas p-Akt expression alterations occurred after day 7. These molecular arrangements can be related to PCa progression but in a longer time when biochemical recurrence. Finally, this report offers important support to the concept that an evaluation of genomic and phenotypic alterations as a consequence of therapy could help to identify subsets of patients who will benefit or be refractory to additive personalized therapies.

\section{Materials and methods}

\section{Patient cohort}

A cohort of 150 patients with intermediate- to high-risk $\mathrm{PCa}$ was retrospectively studied. These tumors were obtained by radical prostatectomy performed at the University of L' Aquila. Out of the 150 patients, 81 received preoperative bicalutamide $(150 \mathrm{mg} /$ day $)$ therapy for 120 days. The remaining 69 patients were untreated (hormone naive). Table 1 summarizes the clinico-pathological characteristics of the 150 patients. Our institutional review board approved the protocol and written informed consent was obtained from all human subjects.

\section{Reagents}

Antibodies against AR and Ki67 were purchased from Santa Cruz Biotechnology Inc (Santa Cruz, CA, USA). Antibodies against phosphorylated forms of EGFR ((pY1086) rabbit polyclonal antibody) and HER2 ((pY1248) clone PN2A) were obtained from Invitrogen (Invitrogen, Carlsbad, USA). Her2 evaluation was performed using HerceptTest (Dako Corp., Glostrup, Denmark). We have selected antibodies against phosphorylated forms of EGFR, Her2, and Akt which were appropriately validated by literature, and we used the recommended controls (positive and negative) according to the manufacturer's instructions. PTEN and p-Akt MABs were purchased from Rockland immunochemicals (Gilbertsville, PA, USA).

\section{LuCaP 35}

The LuCaP 35 xenograft line was developed in the laboratory of one of the authors (Robert L Vesella) and 
Table 1 Patients demography and pathological characteristics

\begin{tabular}{|c|c|c|c|}
\hline Variables & Treated & Untreated & $P$ values \\
\hline Patients & 81 & 69 & \\
\hline Age (year) ${ }^{\mathrm{a}}$ & $67.5 \pm / 5.5$ & $65.2 \pm / 7.9$ & $0.039^{\mathrm{a}}$ \\
\hline \multicolumn{4}{|l|}{ Biopsy Gleason score } \\
\hline Gleason 6 & $44(54.3 \%)$ & $40(58.0 \%)$ & $N S^{b}$ \\
\hline Gleason 7 & $33(40.7 \%)$ & $26(37.7 \%)$ & $N S^{b}$ \\
\hline Gleason 8-10 & $4(4.9 \%)$ & $3(4.3 \%)$ & $N S^{b}$ \\
\hline \multicolumn{4}{|l|}{ Prostatectomy Gleason score } \\
\hline Gleason 6 & NA & $35(50.7 \%)$ & $<0.001^{b}$ \\
\hline Gleason 7 & NA & $30(43.5 \%)$ & \\
\hline Gleason 8-10 & NA & $4(5.8 \%)$ & \\
\hline \multicolumn{4}{|l|}{ Pathological stage } \\
\hline pT2 & $25(30.9 \%)$ & $29(40.0 \%)$ & $N S^{b}$ \\
\hline pT3 & $52(64.2 \%)$ & $35(50.7 \%)$ & $N S^{b}$ \\
\hline pT4 & $4(4.9 \%)$ & $5(7.3 \%)$ & $N S^{b}$ \\
\hline PSA at diagnosis $(\mathrm{ng} / \mathrm{mL})^{\mathrm{a}}$ & $5.9 \pm / 5.4$ & $6.1 \pm / 4.6$ & $N S^{a}$ \\
\hline
\end{tabular}

NA, not assessable.

anpaired student $t$-test.

${ }^{\mathrm{b}} \chi^{2}$ test.

has been previously described (Buhler et al. 1998, Corey et al. 2003). It is passaged by implanting small tissue pieces $(\sim 25 \mathrm{mg})$ subcutaneously into male athymic mice (Balb/c nu/nu ; Charles River Laboratories, Wilmington, MA, USA). LuCaP 35 has a tumor doubling times of 18 days, take rates of $87 \%$, and median prostate specific antigen (PSA) indices (PSA $\left(\mathrm{ng} / \mathrm{ml}\right.$ of serum)/tumor volume $\left.\left(\mathrm{mm}^{3}\right)\right)$ of 0.02 when grown subcutaneously. Forty 6- to 8-week-old CB.17 Fox Chase SCID mice (Charles River Laboratories) were implanted subcutaneously with LuCaP 35 tumor bits (passage 49) as above. When tumors reached 200$400 \mathrm{mg}$, the animals were castrated. Five animals each were killed at the time of castration (day 0 ) and at days 1 , 5 , and 7 post-castration. Tissues were collected and paraffin embedded for the immunohistochemical evaluations that were performed blindly and scored as below.

\section{Immunohistochemistry}

Paraffin sections $(5 \mu \mathrm{m})$ were deparaffinized, blocked, and processed for immunohistochemical detection of PTEN, p/Akt, EGF-R, and Her2/neu using standard techniques, including an avidin-biotinylated peroxidase complex immunoreactivity (IR) for the different markers was detected on $4 \mu \mathrm{m}$ tissue sections cut and evaluated blindly by two pathologist (R P and L V).

a) PTEN immunoreactivity (IR) was scored according to the following formula: staining index $(\mathrm{SI})=($ cytoplasmic staining intensity $\times$ proportion of immunopositive tumor area). PTEN IR was scored as follows: cytoplasmatic staining intensity
(0-3) and the proportion of immunopositive tumor cells $(\leq 10 \%=1 ; 10-50 \%=2 ; \geq 50 \%=3)$ with a PTEN SI ranging between 0 and 9 and a PTEN index of $\leq 4$ indicating a low expression.

b) pAkt IR was scored in agreement with Malik et al. (2002) as follows: 0, none of the cells were stained; 1, weak; 2, moderate; and 3, strong staining intensity. An immunohistochemistry (IHC) score of $2+$ or greater was considered as high expression for p-Akt. Positive controls were breast carcinoma tissues, whereas normal breast tissues were used as negative controls as recommended.

c) EGFR IR was scored according to the following formula: $S I=$ (cytoplasmic staining intensity $X$ proportion of immunopositive tumor) and ranging between 0 and 9. EGFR-specific immunoreactivity was scored as follows: cytoplasmic staining intensity $(0$, no staining; $1+$, faint, incomplete membranous pattern; $2+$, moderate, complete membranous pattern; and $3+$, strong membranous pattern) and the proportion of immunopositive tumor cells $(0$, no immunoreactive cells; $\leq 15 \%=1,16-50 \%=2$, and $>50 \%=3$ immunoreactive cells respectively) with an EGFR $\mathrm{SI} \geq 4$ being considered high expression.

d) HER2/neu IR was scored as follows: 0, no staining; $1+$, faint staining, incomplete membranous pattern; $2+$, moderate, complete membranous pattern; and $3+$, strong membranous pattern. An IHC score of $2+$ or greater was considered as high expression for Her2/neu.

The expression for phosphorylated forms of Her2 and EGFR was scored in the same manner. 
We use as positive controls ovarian cancer tissue for p-EGFR and breast carcinoma tissues or p-Her2 as recommended. Negative controls were tissues from MCF-7 xenografts for p-EGFR and breast carcinoma tissues for $\mathrm{p}$-Her2.

e) Ki67 labeling index was determined by counting 500 cells and determining the percentage of cells staining positively for Ki67.

f) AR expression was determined as percentage of tumor-positive cells with nuclear AR immunoreactivity.

\section{Statistical analysis}

Statistical analysis was performed using SPSS 11.0 (SPSS Inc., Chicago, IL, USA) software. All $P$ values $<0.05$ were considered to indicate significance. All statistical tests were two-tailed. Descriptive statistics were presented as mean and S.D. or $95 \%$ confidence interval $(95 \%$ CI) when appropriated. Continuous variables were analyzed using an unpaired Student's $t$-test. Differences among categorical variables were compared with $\chi^{2}$ test or Fisher's exact test when the tables were too sparse. When we performed multiple comparisons, we used a Bonferroni test with all $P$ values $<0.017$ considered to indicate significance. Multiple logistic regression was performed to determine the strength of relationship among p-Akt, PTEN, EGFR, and Her2 expressions.

\section{Results}

The selected cohort of patients were well matched for clinical and pathological characteristics such as preoperatory PSA, histological Gleason scores, and pathological stages except for age as shown in Table 1.

\section{PTEN expression}

As described in the Materials and methods section, IR data were organized into four intensity levels as shown in Fig. 1 in which we show, in particular, the following patterns: A) the tumor showed a strong, $3+$ PTEN cytoplasmatic staining; B) tumor showed a moderate, $2+$ PTEN cytoplasmic staining; $C$ ) the tumor showed a low/faint, 1+ PTEN cytoplasmic staining; and D) the tumor was negative, 0 cytoplasmic staining. Overall, 70 out of $150(46.7 \%)$ samples presented a uniform $2+, 3+$ staining, 37 out of 90 showed a mixed staining with a moderate and faint profile, and 50 out of $150(33.3 \%)$ were completely negative. For statistical purposes, we considered the score of $<4$ as discriminant between high $(>4)$ and low expressions $(\leq 4)$. Therefore, we observed that 59 out of 150 $(39.3 \%)$ cases presented a score $<4$.

\section{Correlation with Gleason grade}

Tumors were characterized according to the tissue disorganization and invasion criteria of Gleason and broadly grouped into three categories for statistics. Group I consisted of well-differentiated tumors showing little tumor disorganization and limited stromal invasion (Gleason scores of 2-6); group II consisted of less well-differentiated tumors with low to moderate disorganization and a modest degree of stromal invasion (Gleason scores of 7); and group III consisted of poorly differentiated tumors showing high levels of disorganization and stromal invasion (Gleason of 8-10). Considering that the Gleason grade is usually overestimated following androgen ablation treatment, we analyzed our data only for the control group. We observed that PTEN inactivation was related to histological appearance although in a non-significant manner, i.e. significant inactivation of PTEN (low score (4)) in tumors with higher Gleason grades $(P=0.025)$ as shown in Table 2 .

\section{Correlations with pathological stage}

Moreover, we demonstrated a significant reduction of PTEN expression with high pathological stages $(P=0.003)$ as demonstrated in Table 3.

\section{Effects of bicalutamide therapy}

We observed that 20 out of $69(29 \%)$ and 39 out of 81 $(48.1 \%)$ cases in the untreated and treated groups respectively presented a score of 4 . These differences were statistically significant $(P=0.026$, Table 4$)$.

\section{Phospho-Akt expression}

Immunohistochemical pictures of pAkt IR are shown in Fig. 1E-H. The immunostaining for p-Akt can be completely negative (no IR, panel E); weak IR in the cytoplasms and/or membranes (panel F); strong membrane IR (panel G); and strong nuclear IR (panel $\mathrm{H})$. We observed that elevated membrane/nuclear IR (score $2+, 3+$ ) was evident in 67 out of 150 cases of PCa.

\section{Correlation with Gleason grade}

We observed that Akt activity was significantly related to the histological appearance of tumor, i.e., being extremely low in low Gleason grades $(P=0.003)$ as shown in Table 2. 

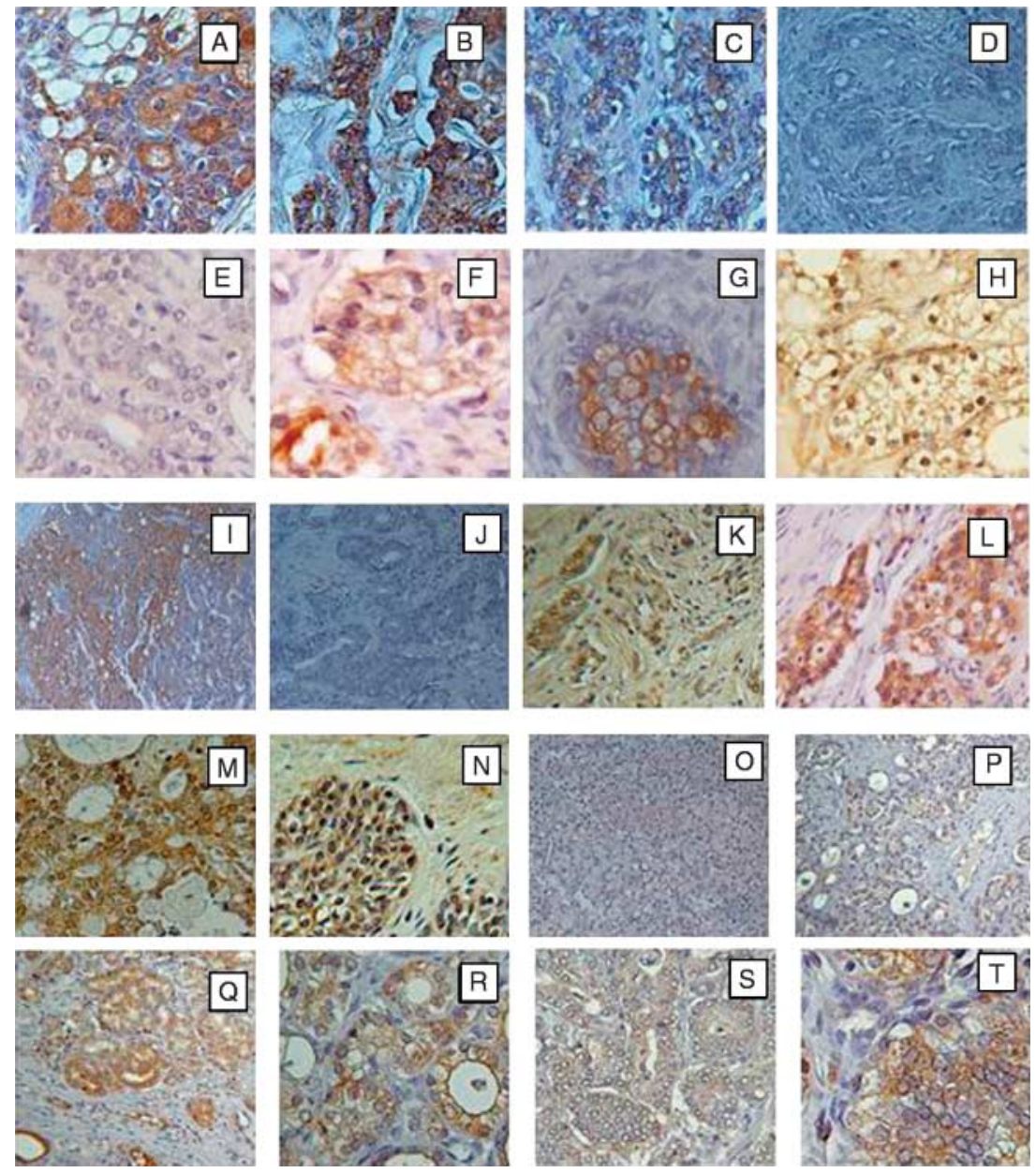

Figure 1 (i) PTEN expression in human PCa tissues (I-L) showing strong (I-J, score 3), moderate (K, score 1), and weak or absent (L, score 0) immunoreactivity. The PCa cases were initially divided into three groups: (a) positive (the entire tumor showed moderate to strong, $2+$ to $3+$, staining); (b) mixed (both positive and negative cells/glands were present); and (c) negative (no staining was seen in the represented tumor). Heterogeneous staining of the tumors was present. Out of 119,50 $(42.0 \%)$ were positive and $27(22.7 \%)$ showed a mixed staining pattern with pictures with moderate (score $2+$ ) and weak (score $1+)$ pattern. Specifically, there were areas of tumor that stained positively, whereas other areas of tumor showed negative staining. The remaining 42 tumors $(35.3 \%)$ were negative. (ii) Akt phosphorylated form expression in the human PCa tissues $(E-H)$ showing absent $(E)$, moderate cytoplasmatic $(F)$, strong membranous $(G)$, and strong nuclear $(H)$ immunoreactivity. (iii) EGFR/Her1 expression in human PCa tissues (I-L) showing heterogeneous immunoreactivity (I). EGFR immunoreactivity was absent or with faint cytoplasmic and membranous staining in a very small $(<10 \%$, score 0 , J) population of tumor, weak to moderate as incomplete membranous staining (present in more than $10 \%$ of tumor cells, score 1 ), moderate as incomplete membranous staining (present in more than 10\% of tumor cells, score 2) and strong as complete membranous staining (present in more than $10 \%$ of tumor cells, score 3). Panel K shows moderate EGFR expression, whereas panel L show strong EGFR expression. (iv) EGFR/Her1 phosphorylated form expression (M and $\mathrm{N}$ ) showing similar pattern when compared with total Her1 expression. (v) Her2 expression in human PCa tissues (M-P) showing a strong (M), moderate (N), weak $(O)$, and absent $(P)$ staining. Her2 IR was absent (score 0), weak to moderate as incomplete membranous staining (present in more than $10 \%$ of tumor cells, score 1), moderate as complete membranous staining (present in more than 10\% of tumor cells, score 2), and strong as complete membranous staining (present in more than $10 \%$ of tumor cells, score 3 ). (v) Her2 phosphorylated form expression $(\mathrm{Q}-\mathrm{T})$ showing similar pattern when compared with total Her2 expression. Panels $\mathrm{A}-\mathrm{H}$, $\mathrm{K}, \mathrm{L}$, and $\mathrm{M}-\mathrm{T}$ represent $400 \times$ images; panels $I-J$ are $100 \times$.

\section{Correlations with pathological stage}

Moreover, we demonstrated a significant increment of Akt activity with high pathological stages $(P=0.002)$ as demonstrated in Table 3.

\section{Effects of bicalutamide therapy}

We observed that 11 out of $69(16 \%)$ and 37 out of 81 $(45.7 \%)$ cases in the untreated and treated groups respectively presented elevated p-Akt expression. 
Table 2 Expressions of phosphatase and tensin homolog deleted on chromosome 10 (PTEN), p-Akt, EGFR, p-EGFR, Her2, and p-Her2 and correlation with the Gleason grade

\begin{tabular}{lccccccc}
\hline Gleason grade & Patients & PTEN & p-Akt & EGFR & p-EGFR & Her2 & p-Her2 \\
\hline $2-6$ & 35 & $6(17.1 \%)$ & $3(8.6 \%)$ & $5(14.3 \%)$ & $4(11.4 \%)$ & $5(14.3 \%)$ & $5(14.3 \%)$ \\
7 & 30 & $11(36.7 \%)$ & $5(16.7 \%)$ & $10(33.3 \%)$ & $8(26.7 \%)$ & $7(23.3 \%)$ & $5(16.7 \%)$ \\
$8-10$ & 4 & $3(75 \%)$ & $3(75 \%)$ & $3(75 \%)$ & $3(75 \%)$ & $3(75 \%)$ & $3(75 \%)$ \\
$P$ values $^{a}$ & & $P=0.025$ & 0.003 & 0.016 & 0.010 & 0.020 & 0.012 \\
\hline
\end{tabular}

It has been chosen to consider PTEN loss (score 4) and overexpressions of p-Akt (score $2+, 3+$ ), EGFR/pEGFR (score 4), and Her2/pHer2 (score $2+, 3+$ ) on 69 untreated patients as indicated in Materials and methods.

${ }^{\mathrm{a}} \chi^{2}$ test.

These differences were statically significant $(P<0.001$, Table 4).

\section{EGFR expression}

EGFR staining in primary tumors shows a strong heterogeneity both among different samples and different areas of the same section (Fig. 1I). In Fig. 1J-L, we show three different EGFR IR pattern: absent (panel $1 \mathrm{~J}$ ), moderate (panel $1 \mathrm{~K}$ ), and strong (panel 1 L) IR.

\section{Correlation with Gleason grade}

We observed that EGFR expression was significantly related to the histological appearance of the tumor, i.e., being significantly lower in low Gleason scores $(P=0.016)$ as shown in Table 2.

\section{Correlations with pathological stage}

Moreover, we demonstrated a non-significant increment of EGFR expression with high pathological stages $(P=0.021)$ as demonstrated in Table 3.

\section{Effects of bicalutamide therapy}

We observed that 18 out of $69(26 \%)$ and 40 out of 81 $(49.4 \%)$ cases in the untreated and treated groups respectively presented elevated EGFR expression. These differences were statically significant $(P=0.006$, Table 4).
In addition, we performed immunodetections against the activated/phosphorylated form of EGFR (Fig. $1 \mathrm{M}$ and $\mathrm{N}$ ) and demonstrated that when EGFR was overexpressed, this receptor was also activated. The IR for p-EGFR was very similar to those observed for total EGFR with similar statistical considerations such as strong correlation with Gleason score (Table 2), pathologic stage (Table 3), and the increased expression after bicalutamide therapy (Table 4).

\section{Her2 expression}

In Fig. 1O-R, we show different Her2 IR patterns: absent (score 0, panel $1 \mathrm{P}$ ), weak (score $1+$, panel $1 \mathrm{P}$ ), moderate (score $2+$, panel $1 \mathrm{Q}$ ), and strong (score $3+$, panel $1 \mathrm{R}) \mathrm{IR}$.

\section{Correlation with Gleason grade}

We observed that Her2 expression was significantly lower in low Gleason scores $(P=0.020)$ as shown in Table 2.

\section{Correlations with pathological stage}

Moreover, we demonstrated a significant increment of Her2 expression with high pathological stages $(P \leq 0.001)$ as demonstrated in Table 3.

\section{Effects of bicalutamide therapy}

We observed that 15 out of $69(21.7 \%)$ and 35 out of 81 $(43.2 \%)$ cases in the untreated and treated group

Table 3 Expressions of phosphatase and tensin homolog deleted on chromosome 10 (PTEN), p-Akt, EGFR, p-EGFR, Her2, and p-Her2 and correlation with the pathological stage

\begin{tabular}{lccccccc}
\hline Stage & Patients & PTEN & p-Akt & EGFR & p-EGFR & Her2 & p-Her2 \\
\hline pT2a/T2b/T2c & 54 & $16(29.6 \%)$ & $8(14.8 \%)$ & $16(29.6 \%)$ & $13(24.1 \%)$ & $7(13.0 \%)$ & $5(9.3 \%)$ \\
pT3a/T3b & 87 & $35(40.2 \%)$ & $35(40.2 \%)$ & $35(40.2 \%)$ & $32(36.8 \%)$ & $36(41.4 \%)$ & $36(41.4 \%)$ \\
PT4 & 9 & $8(88.9 \%)$ & $5(55.5 \%)$ & $7(77.7 \%)$ & $7(77.7 \%)$ & $7(77.7 \%)$ & $5(55.5 \%)$ \\
v values $^{\mathrm{a}}$ & & 0.003 & 0.002 & 0.021 & 0.006 & $<0.001$ & $<0.001$ \\
\hline
\end{tabular}

It has been chosen to consider PTEN loss (score 4) and overexpressions of p-Akt (score $2+, 3+$ ), EGFR/pEGFR (score $\geq 4$ ), and Her2/pHer2 (score 2+, $3+$ ) as indicated in Materials and methods.

a $\chi^{2}$ test. 
Table 4 Expressions of phosphatase and tensin homolog deleted on chromosome 10 (PTEN), p-Akt, EGFR, p-EGFR, Her2, and p-Her2 in untreated and bicalutamide-treated patients

\begin{tabular}{lccccccc}
\hline Therapy & Patients & PTEN & p-Akt & EGFR & p-EGFR & Her2 & p-Her2 \\
\hline UNT & 69 & $20(29 \%)$ & $11(15.9 \%)$ & $18(26.1 \%)$ & $15(21.7 \%)$ & $15(21.7 \%)$ & $13(18.9 \%)$ \\
NHT & 81 & $39(48.1 \%)$ & $37(45.7 \%)$ & $40(49.4 \%)$ & $37(45.6 \%)$ & $35(43.2 \%)$ & $33(40.7 \%)$ \\
$P$ values $^{a}$ & & 0.026 & $<0.001$ & 0.006 & 0.004 & 0.009 & 0.006 \\
\hline
\end{tabular}

It has been chosen to consider PTEN loss (score $<4$ ) and overexpressions of p-Akt (score $2+, 3+$ ), EGFR/pEGFR (score $\geq 4)$, and Her2/pHer2 (score 2+, $3+$ ) as indicated in Materials and methods.

${ }^{\mathrm{a}} \chi^{2}$ test.

respectively presented elevated Her2 expression. These differences were statically significant $(P=0.009$, Table 4$)$. In addition, we performed immunodetections against the activated/phosphorylated form of Her2 and demonstrated that when Her2 was overexpressed, this receptor was also activated. The IR for phospho-Her2 was very similar to those observed for total Her2 with strong correlation with Gleason grade (Table 2), pathologic stage (Table 3), and increased expression after bicalutamide therapy (Table 4).

\section{Relationship among p-Akt and PTEN, EGFR, and Her2}

We observed a high relationship between p-Akt presence and PTEN reduction in the untreated (UNT) group with an $\mathrm{OR}=0.036(95 \% \mathrm{CI}=0.003-0.402$, $P=0.007)$. This suggests that in the presence of low PTEN expression, there was a 27.7-fold increased probability to have high p-Akt expression. When we analyzed the relationship between p-Akt and EGFR expressions, we found an $\mathrm{OR}=20.3$ (95\% $\mathrm{CI}=8.5-35.3, P=0.006$ ), indicating that in presence of high EGFR expression, there was a 20.3-fold increased probability to have high p-Akt expression. Conversely, no statistically significant association was found for Her2 expression with an $\mathrm{OR}=2.1$ (95\% $\mathrm{CI}=0.2-10.6, P=0.537)$. This might suggest that in hormonal untreated patients, PTEN reduction and EGFR overexpression participate to maintain elevated levels of p-Akt, whereas Her2 seems not to have a critical role in this phenomenon.

The multiple logistic regression analysis performed in the bicalutamide-treated patient group suggested that Her 2 overexpression played a critical role in the maintenance of elevated levels of p-Akt, whereas EGFR and PTEN seemed to have secondary roles in this phenomenon. Indeed, we found a very strong association between p-Akt and Her overexpressions with an $\mathrm{OR}=40.5(95 \% \mathrm{CI}=22.3-70.1, P<0.001)$, indicating that in the presence of high Her2 expression, there was a 40.5-fold increased probability to have high p-Akt expression. Conversely, when we analyzed the relationship between p-Akt and low PTEN expressions, we found an $\mathrm{OR}=0.17 \quad(95 \%$ $\mathrm{CI}=0.068-0.162, P<0.001)$, indicating that in presence of low PTEN expression, there was a 5.9-fold increased probability to have high p-Akt expression. Similarly, when we analyzed the relationship between p-Akt and high EGFR expressions, we found an $\mathrm{OR}=5.6$ (CI 95\% $=2.1-14.6, P<0.001$ ), indicating that in the presence of low PTEN expression, there was a 5.6-fold increased probability to have high p-Akt expression.

\section{LuCaP 35 model}

To evaluate whether these molecular arrangements were early events, we used an in vivo preclinical $\mathrm{PCa}$ model, the LuCaP 35 xenograft line. Mice with LuCaP 35 tumors were surgically castrated and killed at different times (Corey et al. 2003). For this purpose, we defined 7 days of castration as short term and evaluated the changes in the molecular and phenotypic profiles at days $0,1,5$, and 7 and after androgen recurrence. The levels of p-Akt were not modified within the 7-day period after castration. PTEN and EGFR expressions were indeed reduced. Conversely, a biphasic trend of Her2 expression was observed with an initial decrement and a restoring of expression at 7 days of castration. However, androgen recurrent tumors presented elevated levels of EGFR, Her2, and p-Akt being completely negative for PTEN.

\section{Discussion and conclusions}

Anti-androgen monotherapy in the form of bicalutamide ( $150 \mathrm{mg}$ /day) is emerging as an alternative in the treatment of locally advanced prostate cancer because of its comparative benefits in relation to quality-of-life issues and associated morbidity. However, a significant number of these patients will recur and need second-line therapies. In addition, the knowledge of molecular events 
induced by the primary therapy may be informative as to the selection and predicted success of subsequent therapies. Herein, we explore such a scenario for the first time following bicalutamide therapy. Molecular and phenotypic profiling in this manner may become an important aspect of personalized therapies. For example, knowledge of alterations in molecular profiling after primary chemotherapy may help explain the lack of significant effects of anti-EGFR tyrosine kinase inhibitors, gefitinib or erlotinib, in the majority of non small lung carcinoma (NSCL) and hormone recurrent prostate carcinoma (HRPC) (Canill et al. 2005) patient treatment. An attractive characteristic of this study is that preoperative neoadjuvant therapy offered a clinical opportunity in which to evaluate the specific molecular effects of bicalutamide therapy and the timeframe in which these events occurred. These events are most likely part of a more complex adaptative mechanism that circumvents androgen deprivation therapy.

The cell populations which remain after an antihormone therapy could be distinct from those that emerge after recurrence. This cell population which arises could originate either by a clonal selection of a resistant clone or by epigenetic mechanisms. Both events could be present together. However, in this short term, the second mechanism could be more likely. In fact, we demonstrated that bicalutamide treatment can induce molecular arrangements in PCa tumors when administered in a relatively short-term therapy like NHT that could explain the probable resistance to bicalutamide that is possible after anti-hormonal treatment. However, these information could be essential for the second-line therapy chosen.

Based on these considerations, we analyzed the expressions of p-Akt, PTEN, EGFR, p-EGFR, Her2, and $\mathrm{p}$-Her2 before and after bicalutamide treatment.

PTEN inactivation is able to modulate the activation of PI3K/Akt-mediated signaling (Whang et al. 1998, Wu et al. 1998), which has been associated with tumor progression (Shukla et al. 2000, Bertram et al. 2006, Verhagen et al. 2006), pharmacological resistance against chemotherapeutics (Faridi et al. 2003, Lee et al. 2004), and anti-target drugs (Han et al. 2006). Constitutive activation of the PI3K/Akt cascade in combination with loss of PTEN has been commonly observed in prostate cancer and results in uncontrolled cell proliferation and reduced apoptosis (Moscatello et al. 1998). In addition, it has been shown that PTENpositive prostate cancer cells might develop high levels of Akt through the activation of several growth factor receptors, such as EGFR and HER2. Targeting of these two receptors in preclinical studies has shown promise.
In this paper, we suggest for the first time that bicalutamide may favor a switch in the control of Akt activation. Indeed, we observed that a subset of hormonal naive patients present elevated p-Akt tumor levels (about 16\%). We also found that about $30 \%$ of patients had a reduction of PTEN, whereas EGFR and Her2 were expressed at high levels in 26.1 and $21.7 \%$ of the patients respectively. When overexpressed, these receptors are also activated since their phosphorylated forms were increased. We found a strict inverse correlation between p-Akt and PTEN levels $(\mathrm{OR}=0.036, P=0.007)$, while a direct correlation was found for $\mathrm{p}$-Akt versus EGFR $(\mathrm{OR}=20.3$, $P=0.006$ ). This results in approximately a $27.1-$ and 20.3-fold increased probability to have high p-Akt levels in presence of low PTEN and high EGFR expressions respectively. Surprisingly, we found no significant relationship between p-Akt and Her2. Thus, these analyses seem to suggest that in hormonal untreated tumors, p-Akt modulation can principally be under PTEN and EGFR control.

After bicalutamide therapy, we observed a significant increase in the percentage of tumors with high p-Akt expression (45.7\%). Also, about $48 \%$ of patients had a reduction of PTEN levels with a significant increase in the percentage of EGFR/p-EGFR- and Her2/p-Her2-positive tumors. Multiple logistic regression analyses suggested that in bicalutamidetreated tumors, p-Akt levels modulation was principally shifted under Her 2 control. In this regard, we found a strong correlation between p-Akt and Her2 levels $(\mathrm{OR}=40.5, P<0.001)$. In addition, we found that PTEN and EGFR had similar importance in the maintenance of elevated p-Akt expression, although at a lower extent $(\mathrm{OR}=0.17$ and $\mathrm{OR}=5.6$ respectively $)$ when compared with Her2. These data indicated a 5.9and 5.6-fold increased probability of having elevated levels of p-Akt in the presence of low PTEN and high EGFR expression levels respectively.

This phenomenon represents a very interesting event since it suggests that the increased levels of Her2 associated with its increased control of Akt activity could reduce the beneficial effects of a second-line therapy as observed in breast cancer (Learn et al. 2005). Learn et al. demonstrated that the levels of Her2 are considered as negative predictors for subsequent paclitaxel therapy.

Our studies also demonstrated that these molecular and phenotypic variations occur at various time points after exposure to the initiating even. These observations arose from our studies using the $\mathrm{LuCaP} 35$ xenograft model and castration as the initial therapy. 
Downregulation of PTEN, EGFR, and Her2 were noted by 1-day post-castration, whereas elevations in p-Akt took longer than 7 days to materialize. Increased expressions of akt activity (p-Akt IR), EGFR, and Her2 associated with loss of PTEN was associated with recurrent $\mathrm{LuCaP} 35$ tumors.

The value of incorporating molecular profiling into the regime of personalized therapy can be already gleaned from the literature (Han et al. 2006). For example, there are suggestions that patients with high HER2 expression, in the presence of androgen receptor (AR) blockade, represent a subgroup of patients at high risk of developing a multi-drug resistant disease (Taplin et al. 2001, Syed 2003, Shi et al. 2004). On the basis of the experiments with androgen-dependent and AI PCa (Agus et al. 1999, 2007, Yano et al. 2006), one would suggest that Her2 inhibitors might be a useful subsequent therapeutic approach in this subset of patients.

From our bicalutamide studies, the presence of a second subset of patients after treatment, in which the maintenance of elevated levels of p-Akt can be due primarily to increased expression/activation of EGFR, suggests the use of EGFR inhibitors in these patients. In addition, some reports demonstrated that the effectiveness of an EGFR tyrosine kinase inhibitor, such as gefitinib, was reduced in presence of elevated levels of Her2 (Cappuzzo et al. 2003, Han et al. 2006, Yokoyama et al. 2006). This might indicate that subjects with high HER2/neu tumor levels might respond worse to anti-EGFR inhibitors. There may be an interesting biological association between the role of HER2/neu and response to treatment with gefitinibbased regimen. Additional observations suggest that an elevated p-Akt activity due to PTEN inactivation is a negative predictor of effectiveness for gefitinib treatment (She et al. 2003, Festuccia et al. 2005b). Thus, the subset of patients that are favorable candidates for gefitinib should have high PTEN, p-Akt, and EGFR, and low Her2 expressions. These considerations should be interpreted cautiously until more robust studies are performed. The presence of elevated EGFR in presence of PTEN may represent a candidate subset of patients in which EGFR tyrosine kinase inhibitor can be satisfactorily used (Parra et al. 2004).

All signaling pathways analyzed in our study suggest that Akt activity is a crucial mediator of effects of EGFR, Her2, and PTEN loss both in naive and hormone-treated tumors. This renders it as an attractive pharmacological target. Some preclinical and clinical studies have already indicated that direct Akt inhibition or the blockade of downstream molecules to Akt (i.e., mTOR) might represent good therapeutical approaches in several resistant malignancies (Wu et al. 1998, 2005, Kristof et al. 2005).

In conclusion, our report suggests that after short-term bicalutamide treatment, EGFR and Her2 expressions are increased, whereas PTEN expression is reduced. This is associated with increased Akt activation and a switch of its control from PTEN/EGFR to Her2/dependent pathways. This could impact the effectiveness of second-line therapies. We suggest that knowledge of the molecular and phenotypic changes in a tumor after hormone therapy could help in the development of new adjuvant anti-target therapies.

\section{Acknowledgment}

The authors declare that there is no conflict of interest that would prejudice the impartiality of this scientific work.

\section{References}

Agus DB, Scher HI, Higgins B, Fox WD, Heller G, Fazzari M, Cordon-Cardo C \& Golde DW 1999 Response of prostate cancer to anti-Her-2/neu antibody in androgen-dependent and -independent human xenograft models. Cancer Research 59 4761-4764.

Agus DB, Sweeney CJ, Morris MJ, Mendelson DS, McNeel DG, Ahmann FR, Wang J, Derynck MK, Ng K, Lyons B et al. 2007 Efficacy and safety of single-agent pertuzumab (rhuMAb 2C4), a human epidermal growth factor receptor dimerization inhibitor, in castration-resistant prostate cancer after progression from taxane-based therapy. Journal of Clinical Oncology 25 675-681.

Ayala G, Thompson T, Yang G, Frolov A, Li R, Scardino P, Ohori M, Wheeler T \& Harper W 2004 High levels of phosphorylated form of Akt-1 in prostate cancer and nonneoplastic prostate tissues are strong predictors of biochemical recurrence. Clinical Cancer Research 10 6572-6578.

Bertram J, Peacock JW, Fazli L, Mui AL, Chung SW, Cox ME, Monia B, Gleave ME \& Ong CJ 2006 Loss of PTEN is associated with progression to androgen independence. Prostate 66 895-902.

Buhler KR, Corey E, Stray JE \& Vessella RL 1998 Study of free and complexed prostate-specific antigen in mice bearing human prostate cancer xenografts. Prostate $\mathbf{3 6}$ 194-200.

Canil CM, Moore MJ, Winquist E, Baetz T, Pollak M, Chi $\mathrm{KN}$, Berry S, Ernst DS, Douglas L, Brundage M et al. 2005 Randomized phase II study of two doses of gefitinib in hormone-refractory prostate cancer: a trial of the National Cancer Institute of Canada-Clinical Trials Group. Journal of Clinical Oncology 23 455-460. 
Cappuzzo F, Gregorc V, Rossi E, Cancellieri A, Magrini E, Paties CT, Ceresoli G, Lombardo L, Bartolini S, Calandri C et al. 2003 Gefitinib in pretreated non-small-cell lung cancer (NSCLC): analysis of efficacy and correlation with HER2 and epidermal growth factor receptor expression in locally advanced or metastatic NSCLC. Journal of Clinical Oncology 21 2658-2663.

Chen ML, Xu PZ, Peng XD, Chen WS, Guzman G, Yang X, Di Cristofano A, Pandolfi PP \& Hay N 2006 The deficiency of Akt1 is sufficient to suppress tumor development in Pten $+/-$ mice. Genes and Development 20 1569-1574.

Corey E, Quinn JE, Buhler KR, Nelson PS, Macoska JA, True LD \& Vessella RL 2003 LuCaP 35: a new model of prostate cancer progression to androgen independence. Prostate 55 239-246.

Craft N, Shostak Y \& Carey M 1999 A mechanism of hormone-independent prostate cancer through modulation of androgen receptor signaling by the HER-2/neu tyrosine kinase. Nature Medicine 5 280-285.

Di Lorenzo G, Tortora G, D’Armiento FP, De Rosa G, Staibano S \& Autorino R 2002 Expression of epidermal growth factor receptor correlates with disease relapse and progression to androgen-independence in human prostate cancer. Clinical Cancer Research 8 3438-3444.

Faridi J, Wang L, Endemann G \& Roth RA 2003 Expression of constitutively active Akt-3 in MCF-7 breast cancer cells reverses the estrogen and tamoxifen responsivity of these cells in vivo. Clinical Cancer Research 9 2933-2939.

Festuccia C, Angelucci A, Gravina GL, Biordi L, Millimaggi D, Muzi P, Vicentini C \& Bologna M $2005 a$ Epidermal growth factor modulates prostate cancer cell invasiveness regulating urokinase-type plasminogen activator activity. EGFreceptor inhibition may prevent tumor cell dissemination. Thrombosis and Haemostasis 93 964-975.

Festuccia C, Muzi P, Millimaggi D, Biordi L, Gravina GL, Speca S, Angelucci A, Dolo V, Vicentini C \& Bologna M $2005 b$ Molecular aspects of gefitinib antiproliferative and pro-apoptotic effects in PTEN-positive and PTENnegative prostate cancer cell lines. Endocrine-Related Cancer 12 983-998.

Gravina GL, Festuccia C, Angelucci A, Poletti A, Capuano D, Vicentini C, Motta M \& Bologna M 2004 Long-term presence of androgens and anti-androgens modulate EGFreceptor expression and MAP-kinase phosphorylation in androgen receptor-prostate positive cancer cells. International Journal of Oncology 25 97-104.

Gross ME, Shazer RL \& Agus DB 2004 Targeting the HERkinase axis in cancer. Seminars in Oncology 31 9-20.

Han SW, Kim TY, Jeon YK, Hwang PG, Im SA, Lee KH, Kim JH, Kim DW, Heo DS, Kim NK et al. 2006 Optimization of patient selection for gefitinib in non-small cell lung cancer by combined analysis of epidermal growth factor receptor mutation, K-ras mutation, and Akt phosphorylation. Clinical Cancer Research 12 2538-2544.
Hermans KG, van Alewijk DC, Veltman JA, van Weerden W, van Kessel AG \& Trapman J 2004 Loss of a small region around the PTEN locus is a major chromosome 10 alteration in prostate cancer xenografts and cell lines. Genes, Chromosomes and Cancer 39 171-184.

Kristof AS, Pacheco-Rodriguez G, Schremmer B \& Moss J 2005 LY303511 (2-piperazinyl-8-phenyl-4H-1-benzopyran-4-one) acts via phosphatidylinositol 3-kinaseindependent pathways to inhibit cell proliferation via mammalian target of rapamycin (mTOR)- and nonmTOR-dependent mechanisms. Journal of Pharmacology and Experimental Therapeutics 314 1134-1143.

Kroger N, Milde-Langosch K, Riethdorf S, Schmoor C, Schumacher M, Zander AR \& Loning T 2006 Prognostic and predictive effects of immunohistochemical factors in high-risk. Clinical Cancer Research 12 159-168.

Learn PA, Yeh IT, McNutt M, Chisholm GB, Pollock BH, Rousseau DL Jr, Sharkey FE, Cruz AB \& Kahlenberg MS 2005 HER-2/neu expression as a predictor of response to neoadjuvant docetaxel in patients with operable breast carcinoma. Cancer 103 2252-2260.

Lee JT Jr, Steelman LS \& McCubrey JA 2004 Phosphatidylinositol $3^{\prime}$-kinase activation leads to multidrug resistance protein-1 expression and subsequent chemoresistance in advanced prostate cancer cells. Cancer Research 64 8397-8404.

Malik SN, Brattain M, Ghosh PM, Troyer DA, Prihoda T, Bedolla R \& Kreisberg JI 2002 Immunohistochemical demonstration of phospho-Akt in high Gleason grade prostate cancer. Clinical Cancer Research 8 1168-1171.

Moscatello DK, Holgado-Madruga M, Emlet DR, Montgomery RB \& Wong AJ 1998 Constitutive activation of phosphatidylinositol 3-kinase by a naturally occurring mutant epidermal growth factor receptor. Journal of Biological Chemistry 273 200-206.

Osman I, Scher HI, Drobnjak M, Verbel D, Morris M, Agus D, Ross JS \& Cordon-Cardo C 2001 HER-2/neu (p185neu) protein expression in the natural or treated history of prostate cancer. Clinical Cancer Research 7 2643-2647.

Parra HS, Cavina R, Latteri F, Zucali PA, Campagnoli E, Morenghi E, Grimaldi GC, Roncalli M \& Santoro A 2004 Analysis of epidermal growth factor receptor expression as a predictive factor for response to gefitinib ('Iressa', ZD1839) in non-small-cell lung cancer. British Journal of Cancer 91 208-212.

She QB, Solit D, Basso A \& Moasser MM 2003 Resistance to gefitinib in PTEN-null HER-overexpressing tumor cells can be overcome through restoration of PTEN function or pharmacologic modulation of constitutive phosphatidylinositol 3'-kinase/Akt pathway signaling. Clinical Cancer Research 9 4340-4346.

Shi Y, Brands FH, Chatterjee S, Feng AC, Groshen S, Schewe J, Lieskovsky G \& Cote RJ 2001 HER2/neu expression in 
prostate cancer: high level of ezpression associated with exposure to hormone therapy and androgen independent disease. Journal of Urology 166 1514-1519.

Shi XB, Ma AH, Tepper CG, Xia L, Gregg JP, GandourEdwards R, Mack PC, Kung HJ \& deVere White RW 2004 Molecular alterations associated with $\mathrm{LNCaP}$ cell progression to androgen independence. Prostate 60 257-271.

Shukla S, Maclennan GT, Marengo SR, Resnick MI \& Gupta S 2005 Constitutive activation of P I3 K-Akt and NF-kappaB during prostate cancer progression in autochthonous transgenic mouse model. Prostate 64 224-239.

Syed S 2003 Combination chemotherapy for hormonerefractory prostate carcinoma: progress and pitfalls. Cancer 98 2088-2090.

Taplin ME, Bubley GJ, Rajeshkumar B, Shuster T, Ko YJ \& Morganstern DE 2001 Docetaxel, estramustine, and shortterm androgen withdrawal for patients with biochemical failure after definitive local therapy for prostate cancer. Seminars in Oncology 4 32-39.

Unni E, Sun S, Nan B, McPhaul MJ, Cheskis B, Mancini MA \& Marcelli M 2004 Changes in androgen receptor nongenotropic signaling correlate with transition of $\mathrm{LNCaP}$ cells to androgen independence. Cancer Research 64 7156-7168.

Uzgare AR \& Isaacs JT 2004 Enhanced redundancy in Akt and mitogen-activated protein kinase-induced survival of malignant versus normal prostate epithelial cells. Cancer Research 64 6190-6199.

Verhagen PC, van Duijn PW, Hermans KG, Looijenga LH, van Gurp RJ, Stoop H, van der Kwast TH \& Trapman J
2006 The PTEN gene in locally progressive prostate cancer is preferentially inactivated by bi-allelic gene deletion. Journal of Pathology 208 699-707.

Whang YE, Wu X, Suzuki H, Reiter RE, Tran C, Vessella RL, Said JW, Isaacs WB \& Sawyers CL 1998 Inactivation of the tumor suppressor PTEN/MMAC1 in advanced human prostate cancer through loss of expression. PNAS 95 5246-5250.

Wu X, Senechal K, Neshat MS, Whang YE \& Sawyers CL 1998 The PTEN/MMAC1 tumor suppressor phosphatase functions as a negative regulator of the phosphoinositide 3-kinase/Akt pathway. PNAS 95 15587-15591.

Wu L, Birle DC \& Tannock IF 2005 Effects of the mammalian target of rapamycin inhibitor CCI-779 used alone or with chemotherapy on human prostate cancer cells and xenografts. Cancer Research 65 2825-2831.

Yano S, Matsuyama H, Hirata H, Inoue R, Matsumoto H, Ohmi C, Miura K, Shirai M, Iizuka N \& Naito K 2006 Identification of genes linked to gefitinib treatment in prostate cancer cell lines with or without resistance to androgen: a clue to application of gefitinib to hormoneresistant prostate cancer. Oncology Reports 15 1453-1460.

Yokoyama H, Ikehara Y, Kodera Y, Ikehara S, Yatabe Y, Mochizuki Y, Koike M, Fujiwara M, Nakao A, Tatematsu M et al. 2006 Molecular basis for sensitivity and acquired resistance to gefitinib in HER2-overexpressing human gastric cancer cell lines derived from liver metastasis. British Journal of Cancer 95 1504-1513. 